\title{
Metabolic Syndrome as a Risk Factor for
}

\section{Sensorineural Hearing Loss in Adult Patients with Turner Syndrome}

This article was published in the following Dove Press journal:

The Application of Clinical Genetics

\author{
Francisco Álvarez-Nava (D) ${ }^{1,2}$ \\ Marcia Racines-Orbe $\mathbb{D}^{3}$ \\ Julia Witt' \\ Jéssica Guarderas $\mathbb{D D}^{\prime}$ \\ Yosselin Vicuña $\mathbb{D}^{3}$ \\ María Estévez ${ }^{4}$ \\ Roberto Lanes (iD) \\ 'Biological Sciences School, Faculty of \\ Biological Sciences, Central University of \\ Ecuador, Quito, Ecuador; ${ }^{2}$ Institute of \\ Genetic Research, University of Zulia, \\ Maracaibo, Venezuela; ${ }^{3}$ Institute of \\ Biomedicine Research, Central University \\ of Ecuador, Quito, Ecuador; ${ }^{4}$ Ecuadorian \\ Foundation in Support of Turner \\ Syndrome, Quito, Ecuador; ${ }^{5}$ Pediatric \\ Endocrine Unit, Hospital De Clinicas \\ Caracas, Caracas, Venezuela
}

Correspondence: Francisco ÁlvarezNava

Biological Sciences School, Faculty of Biological Sciences, Central University of Ecuador, Calle lquique Con Calle Sodiro Number NI4-12I, Parroquia San Blas, Quito, Pichincha 170II3, Ecuador $\mathrm{Tel} / \mathrm{Fax}+593-252-8810$

Email fjalvarez@uce.edu.ec
Background and purpose: Metabolic syndrome (MetS) is a disorder associated with an increased risk of cardiovascular disease. The frequency of each component of MetS in Turner syndrome (TS) subjects is high. An elevated incidence of hearing loss has also been reported in TS. Sensorineural hearing loss (SNHL) affects at least half of young women with TS. The association between MetS and SNHL has not been previously considered in TS. The aim of this study is to evaluate the association between these two conditions.

Patients and Methods: Cross-sectional anthropometric, cardio-metabolic and audiological data were obtained from a cohort consisting of unrelated TS subjects ( $>20$ years of age; $n=$ 93). Metabolic syndrome was defined according to the International Diabetes Federation criteria. Types and severity of hearing loss were based on the American Speech Hearing Association guidelines.

Results: Hearing loss was detected in $74 \%$ of ears from adult TS subjects and SNHL was observed in half of our TS subjects. The prevalence of MetS in TS subjects with or without SNHL was $64 \%$ and $11 \%$, respectively $(\mathrm{P}<0.05)$. After adjusting for age, MetS was related to a ninefold increase in the odds of SNHL. This odds increased in a stepwise manner as the number of MetS components increased.

Conclusion: MetS and its individual components were associated factors for SNHL in TS subjects. A reduction in the number and severity of the components of MetS might potentially contribute to decreasing the progression of SNHL at younger ages, but further studies will be needed to explain the underlying pathological mechanism connecting MetS and SNHL.

Keywords: hearing loss, metabolic syndrome, risk factors, Turner syndrome

\section{Introduction}

Turner syndrome (TS) is a clinical disorder resulting from a partial or total loss of the second sexual chromosome. The severity of the disorder can vary, and clinical features may include short stature, gonadal dysgenesis, often with visible dysmorphic stigmata, and urinary, cardiovascular, skeletal and endocrine abnormalities. ${ }^{1,2}$ This disorder is one of the most common human chromosomal abnormalities, occurring in approximately $1: 2500$ live female births. ${ }^{3}$ In $40 \%$ to $60 \%$ of patients with TS, the karyotypic anomaly is a monosomy $45, \mathrm{X}$, but a variety of other anomalies have been found including mosaicism, $\mathrm{Xp}$ or $\mathrm{Xq}$ deletions, and isochromosome of the $\mathrm{X}$ long arm. ${ }^{1}$ A cell line with a $\mathrm{Y}$ chromosome is also present in $5 \%$ of patients. ${ }^{1}$ The life expectancy in TS is reduced by at least 10 
years ${ }^{2}$ due principally to a threefold increase in the risk of mortality from cardio-cerebrovascular disease. ${ }^{4}$ Insulin resistance and dyslipidemia, both components of the metabolic syndrome (MetS), are the two most common metabolic abnormalities noted in adult patients with TS. ${ }^{5,6}$ Metabolic syndrome is a cluster of common disorders that include visceral adiposity, hypertension, insulin resistance, and dyslipidemia and may lead to the development of Type 2 Diabetes Mellitus (T2DM) and cardiovascular disease. $^{7}$ Although several studies have reported an increased prevalence of each of the MetS components in $\mathrm{TS},{ }^{4,5,8,9}$ few studies have determined the global prevalence of MetS in TS. ${ }^{10,11}$ In addition, most of these studies have methodological deficiencies such as different age ranges. Data on the prevalence of MetS in TS adult patients are still scarce.

An elevated incidence of hearing loss (HL) has been reported in adult patients with TS. ${ }^{12-14}$ This deficiency can be conductive, sensorineural, or mixed. ${ }^{13,15}$ Sensorineural hearing loss (SNHL) is the most prevalent as it affects at least half of the women with TS 35 years or older. ${ }^{16}$ SNHL in TS is gradually progressive, resulting at age 40 in hearing comparable to that of women aged 60 in the general population. ${ }^{12}$ Thus, both MetS and SNHL are comorbidities frequently reported in TS patients with significant effects on their health. In addition, data from several large epidemiological and smaller cohort studies suggest a relationship between HL and MetS in the general population. ${ }^{17-21}$ Microvascular damage has been reported in $\mathrm{SNHL}^{22}$ and has also been found to be a complication of MetS in non-TS patients. ${ }^{18}$ Therefore, MetS may be an associated risk factor for SNHL in adult patients with TS. It is feasible for MetS to appear before the onset and development of SNHL so that it could modify the course and/or severity of SNHL. However, these two conditions could also be comorbidities, so that both could develop at the same time. It is even possible that by modifying cardiovascular risk factors in women with TS, the course and/or severity of SNHL could be reduced, without there being an inevitable causal association between these two conditions. Additionally, fetal programming may be a component that could be taken into account, as it can link these two conditions without a causal association between them. As well as, associated factors to HL, such as the influence of age, chromosomal constitution, effect of the parental origin of the retained X-chromosome, estrogen and rhGH therapies, craniofacial malformations and ear infections have been previously evaluated in TS subjects. ${ }^{12-15,23,24}$ Nevertheless, the effect of such associated factors on HL has been inconsistent and controversial and there is no general agreement of which the main factors affecting HL in TS are. In addition, several studies to date have tended to focus on the influence of one rather than multiple associated factors. There have been, however, no comprehensive studies exploring a relationship between HL and MetS in TS. This study aimed to identify potential associated factors for HL in a cohort of adult TS subjects and to evaluate the association between $\mathrm{HL}$ and MetS in TS.

\section{Materials and Methods}

This was a cross-sectional correlational study carried out in two Latin-American universities over the past 7 years. Ninetythree unrelated adult TS patients $(>20$ years old) were recruited from two sources. Venezuelan subjects $(n=56)$ were periodically examined at the outpatient Medical Genetics clinic of the Genetic Research Institute, University of Zulia, between January 2010 and December 2016. A second group of Ecuadorian individuals $(n=37)$ were recruited through the Ecuadorian Foundation in Support of Turner Syndrome; they were contacted through the use of letters, e-mails and telephone calls and subsequently underwent a comprehensive health examination between January and November 2017. No statistical difference was found between subjects of the two different countries at the beginning of the study with respect to the demographic or genetic data (age, socio-economic status, education level, height-SDS, weight-SDS, BMI-SDS, karyotype distribution). The study group was subdivided into TS subjects with MetS and TS subjects without MetS (control group). A reference group of 57 healthy women, 20 to 49 years of age, matched by age and BMI with the study group, was also included in the study. No individuals presented with organic disease. The diagnosis of TS was established by lymphocyte chromosomal analysis in combination with the presence of typical clinical features. Karyotypes were distributed as follows: 45,X ( $\mathrm{n}=37)$; 45,X/ 46,XX (n=19); karyotypes with Xq-isochromosomes (16) or Xp deletions ( $\mathrm{n}=7$ ); karyotypes with $\mathrm{Y}$ chromosome material $(n=5)$; karyotypes with multiple cell lines $(n=5)$ and karyotypes with a marker or ring chromosome $(n=4)$. None of the subjects received treatment with growth hormone or anabolic steroids.

Information on exposure to estrogen (age at menarche, start of induction of puberty, premature ovarian failure, duration and cessation of hormone replacement therapy (HRT)) was collected. Five of the patients menstruated 
spontaneously. All patients, except six, received conventional HRT. Fifty-three TS subjects (57\%) received HRT for more than 5 years. The remaining 40 subjects were either poorly or irregularly treated (for less than 2years (range: 6 months to 7.3 years) or never received HRT and seven of these patients received HRT for an unspecified period. If a patient was receiving HRT, it was discontinued 4-weeks prior to the assessment.

The study protocol was assessed and approved by the institutional research ethics committees in human subjects of the two participating universities. The procedures and possible discomforts/risks related to the study were fully explained to all subjects before beginning the study and they voluntarily decided to take part in it and signed an informed written consent in front of a witness.

Anthropometric evaluations were performed by trained physicians using standard techniques. Several indices and ratios were then calculated: Body Mass Index (BMI), waist/standing height (WHtR) and waist/hip (WHR) ratios. Following 10 mins of rest blood pressure (Systolic (SBP) and Diastolic Blood Pressure (DBP) was measured in the left arm with an appropriate size cuff and in the sitting position. A $10-\mathrm{mL}$ whole blood sample was then obtained for the measurement of fasting plasma glucose (FPG), total cholesterol, Low-Density Lipoprotein-cholesterol (LDL-c) and High-Density Lipoprotein-cholesterol (HDL-c) and triglyceride (TAG) concentrations by the enzymatic method. Metabolic syndrome was diagnosed using the International Diabetes Federation (IDF) consensus. ${ }^{7}$ According to the new IDF definition, for a woman to be defined as having the MetS they must present: 1) Central obesity (defined as waist circumference $\geq 80 \mathrm{~cm}$ ) plus any two of the following four factors: 2) fasting plasma glucose $5.6 \mathrm{mmol} / \mathrm{L}(100 \mathrm{mg} / \mathrm{dL}) ; 3)$ HDL-c level $<1.29$ $\mathrm{mmol} / \mathrm{L}(<50 \mathrm{mg} / \mathrm{dL}) ; 4) \mathrm{TAG} \geq 1.7 \mathrm{mmol} / \mathrm{L}(>150 \mathrm{mg} /$ $\mathrm{dL}$ ) or specific treatment for this; and 5) blood pressure $\geq 130 / 85 \mathrm{~mm} \mathrm{Hg}$ or treatment of previously diagnosed hypertension.

Following at least $24 \mathrm{hrs}$ of noise avoidance, audiological assessment was performed based on the American Speech Hearing Association (ASHA) guidelines for manual pure tone audiometry. Diagnostic standard pure tone audiometry included measurement of air-conduction thresholds from 0.25 through $8 \mathrm{kHz}$, while the bone conduction thresholds were measured at frequencies 0.5 through $6 \mathrm{kHz}$ for each ear separately with a calibrated two-channel audiometer with earphones (MA 53, Maico Inc.) in a soundproof room in ambient noise of $<45 \mathrm{~dB}$ HL. ${ }^{19,20}$
Conductive hearing (CHL) loss was defined as an air-bone gap of at least $15 \mathrm{~dB}$ HL at one or more frequencies with an air-conduction threshold greater than $25 \mathrm{~dB}$ HL; Mixed Hearing Loss (MHL) was diagnosis when air-conduction thresholds were greater than $20 \mathrm{~dB}$ HL at any frequency in the frequency range $0.25-4 \mathrm{kHz}$, with an air-bone gap of greater than $10 \mathrm{~dB} \mathrm{HL}$ at one frequency at least in the range $0.5-4 \mathrm{kHz}$ at which there was an elevation of air-conduction thresholds; ${ }^{13,14,23}$ SNHL was defined by air-conduction thresholds greater than $25 \mathrm{~dB} H \mathrm{HL}$ at one or more frequencies and the lack of an air-bone gap. The severity classification of SNHL was based on the average of air-conduction thresholds obtained at 500,1000, and $2000 \mathrm{~Hz}$ and SNHL was defined as mild (mSNHL) (26-40 dB HL), moderate (moSNHL) (41-55 dB HL), moderately severe (mosSNHL) (56-70 dB HL), severe (sSNHL) (71-90 dB HL) and profound ( $>90 \mathrm{~dB} \mathrm{HL})$.

Data were analyzed using IBM SPSS Statistics 19.0 (SPSS Inc., Chicago, IL, USA) and GraphPad InStat 7.00 (GraphPad Software, Inc., La Jolla, CA, USA). The general features of the participants are described as number of cases and mean and standard deviations unless otherwise mentioned. Results as determined by $95 \%$ confidence intervals (95\% CI) and a $\mathrm{P}$ value of $<0.05$ were considered to be statistically significant. The prevalence of MetS and different types of hearing loss are indicated as percentages. To compare the proportions of categorical variables, ie, differences in the percentages of MetS and its components between SNHL- and Non-SNHL TS subjects, the two proportion Z-test was used. After checking the normality of the quantitative variables with the Kolmogorov-Smirnoff test ( $\mathrm{P}$ $<0.05$ ), a Student's $t$-test was performed to compare means between groups for continuous variables. Conversely, when the variables did not fit into the criteria of normality they were expressed as median (range) and a Mann-Whitney $U$-test was carried out. Odds ratios, adjusted for age (aOR: adjusted Odds Ratio) along with the corresponding 95\% CI were used to examine the strength of associations between the different types of hearing loss and MetS or its components. Since HL is associated with age, TS subjects were classified into two categories ( $<30$ and $\geq 30$ years of age) so as to establish a proportional interval based on the age limits of our study and reference groups. Binary logistic regression analysis was performed to identify independent accompanying factors for SNHL in TS subjects. First, multiple bivariable logistic regression analyses were carried out including variables for categories of MetS, age, HRT, complete Xp deletion, pterygium colli and otitis media as independent 
variables. Factors associated with SNHL were identified by a marginal statistical significance $P$ value of $<0.2$ using the backward elimination method to arrive at the final univariable model of the predictors of SNHL. However, otitis media was added to the model because it was selected a priori. Then, these independent variables were considered candidate variables and exported to a multivariable binary logistic regression model for controlling the possible effect of confounders. SNHL, dichotomously defined, was the dependent variable. In the final multivariable model, $\mathrm{P}$ value $<0.05$ for two-sided tests was set as the level of significance. The odds ratio (OR) with $95 \%$ confidence interval $(\mathrm{CI})$ was used to test the strength of associations. Nagelkerke pseudo-R2 was used to indicate how well the model explains the data. Goodness of fit for a model was determined using the postregression diagnostics HosmerLemeshow test. An appropriate model was indicated by a non-significant $\chi 2(\mathrm{P}>0.05)$.

\section{Results}

\section{Type, Frequencies and Percentages of Hearing Loss in TS Subjects}

Comparison of the type and frequency of hearing loss between the right and left ear in TS subjects is detailed in Figure 1. No differences in the type of HL between the right and left ears were detected among TS subjects (two proportion Z-test $\mathrm{P}=>0.05$ for all comparisons). Abnormal puretone threshold data were detected in 138 of $186(74.2 \%)$ ears in TS subjects, compared to only $17 \%$ in the reference subjects. In the TS group, CHL was detected in 28 of 186 (15.1\%) ears, while mixed hearing loss (MHL) was found in 18 of $186(9.7 \%)$ ears. Sensorineural hearing loss was present in 92 of $186(50 \%)$ ears. According to severity, mild SNHL was detected in $27.9 \%$ of TS subject ears, while 33 of 186 ears $(17.7 \%)$ showed a moderate degree of SNHL. Moderately severe and severe SNHL comprised $5(2.7 \%)$ and $2(2.2 \%)$ of the ears, respectively. No subjects with TS presented profound SNHL. No differences between right and left ears were detected among TS subjects with SNHL (Figure 1). Thirty-three of 47 TS subjects (64.7\%) presented bilateral SNHL (not necessarily identical in severity).

\section{Associated Factors for Hearing Loss in TS Subjects Cardio-Metabolic Variables}

The clinical, anthropometric and bio-impedance features of the TS study group are listed in Table 1. Women with TS and SNHL were significantly shorter and had higher weight, BMI, waist circumference (WC), WHR, WHtR and SBP and DBP than non-SNHL subjects (Table 1). Being overweight/obese (BMI $\geq 25 \mathrm{~kg} / \mathrm{m} 2$ ) was related with an increased odds of SNHL (6.4; 95 CI: 2.5-16.3). Overweight (BMI $\geq 25<30 \mathrm{~kg} / \mathrm{m} 2$ ) and obesity (BMI $\geq 30 \mathrm{~kg} / \mathrm{m} 2$ ) were associated with a twofold (1.8-5.2) and 10-fold (2.2-47.1) increase in the odds of SNHL, respectively (for two conditions, two proportion Z-test, $\mathrm{P}<$ $0.05)$. With respect to bio-impedance variables, individuals with TS and SNHL showed greater fat mass (FM), percentage of fat mass (PFM) and free fat mass (FFM)/FM (FFM/FM) ratio than non-SNHL TS subjects. No difference between TS subjects with SNHL or without SNHL was observed regarding Total Body Water (TBW) and FFM, whereas Percentage of Body Water (PBW) and
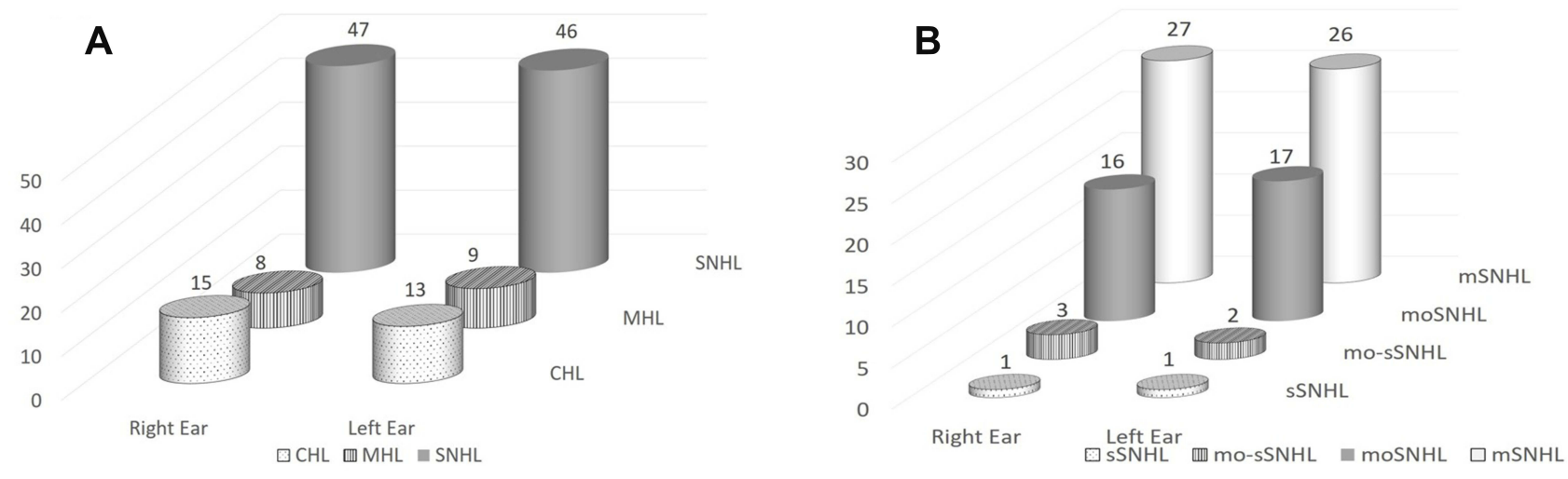

Figure I Number of TS subjects among the different types of hearing loss $(\mathbf{A})$ and distributed according to the severity of SNHL $(\mathbf{B})$. (Two proportion Z-test $\mathrm{P}=>0.05$ for all comparisons).

Abbreviations: $\mathrm{CHL}$, conductive hearing loss; $\mathrm{MHL}$, mixed hearing loss; $\mathrm{SNHL}$, sensorineural hearing loss; $\mathrm{mSNHL}$, mild $\mathrm{SNHL}$; moSNHL, moderate $\mathrm{SNHL}$; mo-sSNHL, moderately severe SNHL; sSNHL, severe SNHL. 
Table I Clinical, Anthropometric and Bio-Impedance Variables in Turner Syndrome Subjects

\begin{tabular}{|c|c|c|c|}
\hline & SNHL & $\begin{array}{l}\text { Non- } \\
\text { SNHL }\end{array}$ & $\mathbf{P}$ \\
\hline Number of Subjects, \% (n) & $50.5(47)$ & $49.5(46)$ & $0.543 \dagger$ \\
\hline Age (Years) & $31.1 \pm 7.1$ & $27.2 \pm 6.3$ & $<0.001$ \\
\hline $\begin{array}{l}\text { Age at Pubertal Induction } \\
\text { (Years) }\end{array}$ & $17.2 \pm 5.2$ & $16.8 \pm 4.1$ & 0.3189 \\
\hline Years of HRT (Years) & $7.91 \pm 6.4$ & $8.2 \pm 5.7$ & 0.2317 \\
\hline Height $(\mathrm{cm})$ & $138.3 \pm 3.1$ & $140.5 \pm 3.5$ & $0.0409 \ddagger$ \\
\hline Weight (kg) & $55.7 \pm 11.3$ & $46.9 \pm 6.3$ & $<0.001$ \\
\hline BMI $\left(\mathrm{kg} / \mathrm{m}^{2}\right)$ & $28.6 \pm 5.8$ & $23.6 \pm 3.4$ & $<0.001$ \\
\hline Waist Circumference $(\mathrm{cm})$ & $83.1 \pm 14.3$ & $72.8 \pm 7.1$ & $<0.001$ \\
\hline Waist/Hip Ratio & $0.90 \pm 0.1$ & $0.9 \pm 0.1$ & 0.014 \\
\hline Waist/Height Ratio & $0.6 \pm 0.1$ & $0.5 \pm 0.1$ & $<0.001$ \\
\hline $\begin{array}{l}\text { Systolic Blood Pressure } \\
(\mathrm{mmHg})\end{array}$ & $\begin{array}{l}138.0 \pm \\
18.0\end{array}$ & $\begin{array}{l}119.0 \pm \\
17.0\end{array}$ & $<0.001$ \\
\hline $\begin{array}{l}\text { Diastolic Blood Pressure } \\
(\mathrm{mmHg})\end{array}$ & $86.0 \pm 14.0$ & $77.0 \pm 13.0$ & $<0.001$ \\
\hline Total Body Water (L) & $24.3 \pm 4.1$ & $24.1 \pm 4.9$ & $0.8826 \ddagger$ \\
\hline $\begin{array}{l}\text { Percentage of Body Water } \\
\text { (\%) }\end{array}$ & $44.9 \pm 9.9$ & $51.4 \pm 8.8$ & 0.002 \\
\hline Fat Mass (kg) & $24.3 \pm 9.6$ & $16.6 \pm 5.3$ & $\begin{array}{l}< \\
0.001 \ddagger\end{array}$ \\
\hline Percentage of Fat Mass (\%) & $41.3 \pm 10.7$ & $34.5 \pm 6.9$ & $\begin{array}{l}< \\
0.001 \ddagger\end{array}$ \\
\hline Free Fat Mass (kg) & $25.3 \pm 7.9$ & $25.5 \pm 9.0$ & 0.9089 \\
\hline Percentage Free Fat Mass (\%) & $46.9 \pm 16.9$ & $54.0 \pm 17.9$ & $0.026 I$ \\
\hline $\begin{array}{l}\text { Free Fat Mass/Fat Mass } \\
\text { Ratio }\end{array}$ & $1.1 \pm 0.6$ & $0.8 \pm 0.4$ & 0.0013 \\
\hline
\end{tabular}

Notes: Data expressed as mean and SD. $\dagger$ : two proportion Z-test. $\mathrm{P}$ indicates difference between SNHL-TS and Non-SNHL-TS subjects calculated by the Student's $t$ test unless otherwise specified ( $\ddagger$ the Mann-Whitney $U$-test). Bold font represents $P$ values which are statistically significant.

Abbreviations: SNHL, sensorineural hearing loss; SNHL-TS, Turner syndrome subjects with SNHL; Non-SNHL-TS, Turner syndrome subjects without SNHL; HRT, hormone replacement therapy; BMI, body mass index.

Percentage of Free Fat Mass (PFFM) were significantly lower in TS subjects with SNHL than in those with nonSNHL (Table 1). Mean HDL-c levels (mg/dL) were similar in TS with and without SNHL (49.9 \pm 12.5 vs $54.9 \pm$ 17.4; $\mathrm{P}=0.2403$, Mann-Whitney test). By contrast, TS subjects with SNHL had significantly increased FPG (87.4 \pm 13.5 vs $80.6 \pm 12.0 ; \mathrm{P}=0.021)$, TAG $(178.6 \pm 56.7$ vs $146.1 \pm 58.5 ; \mathrm{P}=0.0097)$, LDL-c $(155.1 \pm 62.1$ vs $134.8 \pm$ 67.6; $\mathrm{P}=0.01067)$ and total cholesterol (TC) $(222.4 \pm 43.2$ vs $198.9 \pm 56.3 ; \mathrm{P}=0.0294)$ levels $(\mathrm{mg} / \mathrm{dL})$ when compared to TS subjects without SNHL. All atherogenic indexes, including the Castelli Index $(4.7 \pm 1.3$ vs $3.8 \pm$ 1.2; $\mathrm{P}<0.001)$, the TAG/HDLc ratio $(3.9 \pm 1.8$ vs $2.9 \pm$ $1.5 ; \mathrm{P}=0.0045$ ), the Atherogenic Index of Plasma (AIP) $(0.5 \pm 0.2$ vs $0.4 \pm 0.2 ; \mathrm{P}=0.0051)$, LDL-c/HDL-c ratio
$(3.3 \pm 1.4$ vs $2.6 \pm 1.4 ; \mathrm{P}=0.0202)$ and the Non-HDL-c/ HDL-c ratio $(3.7 \pm 1.3$ vs $2.8 \pm 1.2 ; \mathrm{P}<0.001)$ were significantly higher in TS subjects with SNHL than in those without SNHL. Hypertension was significantly more common in TS with SNHL than in TS without SNHL $(\mathrm{P}<0.05)$. No significant differences between TS subjects with and without CHL or with or without MHL were found for anthropometric, cardiometabolic or bioimpedance variables.

The prevalence of the components of MetS in SNHL and in non-SNHL-TS subjects is shown in Table 2. The prevalence of each component of MetS was higher in SNHL than in Non-SNHL-TS subjects or in the reference group. Consequently, the prevalence of MetS was higher in SNHL than in Non-SNHL TS subjects $(63.8 \%$ vs $10.9 \%$, two proportion Z-test $\mathrm{P}=<0.05$ ). In the crude model, the odds ratio of SNHL according to MetS was $10.74,95 \%$ CI $=3.8-30.4$ (Z-statistic: 4.163 and $\mathrm{P}<0.05$ ) (Tables 3 and 4). Since SNHL was shown to be age-dependent, TS subjects were classified into two categories $(<30$ and $\geq 30$ years of age) and after adjusting for age, TS subjects with MetS still had a significantly increased odds of SNHL (aOR = 8.9, 95\% CI $=3.00-26.3$ ) (Table 3). The odds of having SNHL in TS subjects increased in a stepwise manner as the number of MetS components increased. Individuals with TS and four components of MetS had a higher aOR for SNHL than those with three components [11.1 (95\% CI: 2.4-51.6) vs 3.2 (95\% CI: $1.1-10.8)](\mathrm{P}=<0.001)$. None subjects had five components of MetS. No significant differences between TS subjects with or without CHL or with or without MHL were found for the prevalence of MetS or for each component of MetS.

\section{Age}

The mean \pm SD for age was $29.4 \pm 6.7$ (range 20 to 49 ) years for the TS group and $29.8 \pm 4.7$ (range 20 to 49 ) years for the reference group. However, individuals with TS and SNHL were older than TS subjects without SNHL (Table 1). While the frequency of CHL and MHL remained constant across the age range studied (in 10year age increments), a significant linear association between age and percentage of SNHL in TS subjects was detected $(\mathrm{P}<0.001$, chi-squared Mantel-Haenszel test, Table 3). As age increased the proportion of TS subjects with SNHL also increased. The prevalence of SNHL increased from $44.7 \%$ to $60 \%$ from an age of $\geq 29$ to $\leq 39$ years, reaching $66.7 \%$ in TS subjects older than 40 years of age (Supplementary Table). 
Table 2 Prevalence of the Metabolic Syndrome and Its Components in SNHL and in Non-SNHL TS Subjects and Odds Ratios of SNHL According to MetS

\begin{tabular}{|c|c|c|c|c|c|c|}
\hline & \multirow[t]{2}{*}{ SNHL $(n=47)$} & \multirow[t]{2}{*}{ Non-SNHL $(n=46)$} & \multirow[t]{2}{*}{$\mathbf{P}$} & \multirow[t]{2}{*}{ aOR } & \multicolumn{2}{|c|}{$(95 \% \mathrm{Cl})$} \\
\hline & & & & & Lower & Upper \\
\hline MetS \% (n) & $63.8(30)$ & $10.8(5)$ & $<0.05$ & $8.9 \S$ & 3.0 & 26.3 \\
\hline Pathological Waist Circumference \% (n) & $93.8(45)$ & $26.1(12)$ & $<0.05$ & 6.5 & 2.1 & 19.9 \\
\hline Hypertriglyceridemia \% (n) & $85.4(42)$ & $39.1(18)$ & $<0.05$ & 2.9 & 1.5 & 5.6 \\
\hline Low HDL-c \% (n) & $43.8(21)$ & $21.7(10)$ & $<0.05$ & 1.4 & 1.0 & 2.1 \\
\hline Hypertension \% (n) & $37.5(18)$ & $8.7(4)$ & $<0.05$ & 1.8 & 1.3 & 2.5 \\
\hline Hyperglycemia \% (n) & $22.9(11)$ & $4.4(2)$ & $<0.05$ & 1.7 & 1.24 & 2.4 \\
\hline
\end{tabular}

Notes: Comparisons were done by two proportion Z-test. §: unadjusted OR; aOR, estimation of odds ratio of SNHL according to each individual component adjusted for MetS (presence or absence) by the Mantel-Haenszel method.

Abbreviations: MetS, metabolic syndrome; HDL-c, high-density lipoprotein-cholesterol; SNHL, sensorineural hearing loss; Non-SNHL, without SNHL; OR, odds ratio; aOR, adjusted odds ratio; $95 \% \mathrm{Cl}, 95 \%$ confidence intervals.

Table 3 Estimation of Odds Ratios of SNHL According to MetS Adjusted for Age (<30 and $\geq 30$ Years Old)

\begin{tabular}{|c|c|c|c|c|c|c|}
\hline \multirow{2}{*}{\multicolumn{2}{|c|}{$\begin{array}{l}\text { Age of TS } \\
\text { Subjects }\end{array}$}} & \multirow{3}{*}{$\begin{array}{l}\text { SNHL } \\
19\end{array}$} & \multirow{3}{*}{$\begin{array}{l}\text { Non- } \\
\text { SNHL } \\
4\end{array}$} & \multirow{3}{*}{$\begin{array}{l}\text { OR } \\
9.50\end{array}$} & \multicolumn{2}{|c|}{$95 \% \mathrm{Cl}$} \\
\hline & & & & & \multirow{2}{*}{\begin{tabular}{l|} 
Lower \\
2.32
\end{tabular}} & \multirow{2}{*}{\begin{tabular}{|l} 
Upper \\
38.88
\end{tabular}} \\
\hline$<30$ & MetS & & & & & \\
\hline Years & $\begin{array}{l}\text { No } \\
\text { MetS }\end{array}$ & 7 & 14 & & & \\
\hline \multirow{2}{*}{$\begin{array}{l}\geq 30 \\
\text { Years }\end{array}$} & MetS & 10 & 2 & \multirow[t]{2}{*}{11.82} & \multirow[t]{2}{*}{2.22} & \multirow[t]{2}{*}{63.02} \\
\hline & $\begin{array}{l}\text { No } \\
\text { MetS }\end{array}$ & II & 26 & & & \\
\hline \multirow{2}{*}{\multicolumn{2}{|c|}{ Total }} & \multirow[t]{2}{*}{47} & \multirow[t]{2}{*}{46} & 10.74 & 3.80 & 30.40 \\
\hline & & & & $8.9^{*}$ & $3.0 *$ & $26.3^{*}$ \\
\hline
\end{tabular}

Notes: *Estimation of Adjusted Odds Ratio by the Mantel-Haenszel method where the results of the tests of homogeneity from Breslow-Day and Tarone's were 0.664 and 0.665 , respectively, and asymptotic significance (2-sided) of tests of conditional independence by Cochran's and Mantel-Haenszel were 0.00I.

Abbreviations: SNHL, sensorineural hearing loss; MetS, metabolic syndrome; OR, odds ratio; $95 \% \mathrm{Cl}, 95 \%$ confidence intervals $(P<0.00 \mathrm{I}$ for all calculated $\mathrm{OR})$.

\section{Estrogens}

A majority of TS subjects in our cohort received HRT for more than 5 years $(n=53, \approx 60 \%)$. The proportion of SNHL was not significantly different in TS subjects who received HRT $(n=16)$ versus those who did not receive it $(\mathrm{n}=14)(\mathrm{OR}=1.14 ; 95 \% \mathrm{CI}: 0.57-2.31)$ (two proportion Z-test, $\mathrm{P}>0.05$ ), nor did the duration of HRT ( $\geq 5$ years) $(\mathrm{n}=13$ vs $12 ; \mathrm{OR}=1.08,95 \% \mathrm{CI}: 0.50-2.32)$ affect the prevalence of SNHL. Thus, SNHL was not related to previous HRT use.

\section{External Craniofacial Signs}

Anomalies in the derivatives of the first and second pharyngeal arch, including 1) ear anomalies (low-set or cupped ears, deformity of the helix, narrowing of the external auditory canal), 2) dismorphic palate (prominent lateral ridges, narrow and/or high arched palate), 3) pterygium colli, 4) low posterior hair line and 5) micrognathia, were present in 86 $(92.5 \%)$ of the 93 TS subjects. The frequencies, percentages and odds ratio $(95 \% \mathrm{CI})$ of these anomalies in TS subjects with or without SNHL ( $n=47$ vs 46, respectively) can be seen in Figure 2. Sixty-three patients (67.7\%) had ear anomalies; 76 (81.7\%) presented with dismorphic palates; $33(35.5 \%)$ had pterygium coli, $71(76.3 \%)$ had a low posterior hairline and 31 (33.3\%) exhibited micrognathia. The proportion of these five external craniofacial signs were significantly different between TS subjects with or without

Table 4 Multivariable Binary Logistic Regression Analysis Results of Factors Accompanying SNHL Among TS Subjects

\begin{tabular}{|l|l|l|l|l|l|l|l|}
\hline Predictor & Category & B & Wald $\chi^{2}$ & P & \multicolumn{2}{l|}{ Odds Ratio (Exp(B)) } & \multicolumn{2}{|l|}{ 95\% Cl for Exp(B) } \\
\cline { 3 - 7 } & & & & & & Lower & Upper \\
\hline MetS & $\geq 3$ components & 2.374 & 20.007 & $<0.0001$ & 10.741 & 3.795 & 30.396 \\
Age & $\geq 30$ years & -1.18 & 2.82 & 0.093 & 0.309 & 0.078 & 1.218 \\
Complete deletion XP & Yes & -0.514 & 0.559 & 0.454 & 0.598 & 0.155 & 2.301 \\
HRT & $\geq 5$ years & -0.178 & 2.407 & 0.813 & 0.837 & 0.191 & 3.671 \\
Pterygium colli & Yes & 1.701 & 5.22 & $<0.001$ & 5.48 & 3.69 & 18.63 \\
\hline
\end{tabular}

Abbreviations: SNHL, sensorineural Hearing Loss; MetS, metabolic syndrome; HRT, hormone replacement therapy; Exp(B), adjusted odds ratio for binary logistic regression model; $95 \%$ C195\% confidence intervals. 
SNHL (Figure 2). By contrast, a significant increase in the odds of CHL was only found when pterygium colli was present $(\mathrm{OR}=3.0,95 \% \mathrm{CI}=1.2-7.6)$.

\section{Chromosomal Constitution}

In order to obtain an adequate size that allowed for statistical comparison of the data, we divided the TS subjects into two groups of karyotypes: 1) TS subjects with complete deletion of the short arm $(45, X$ and $46, X i(X q)(n=53)$; and 2$)$ TS subjects with other cytogenetic findings including $45, \mathrm{X} / 46$, $\mathrm{XX}$, multiple mosaicism, structural abnormalities (partial Xp or $\mathrm{Xq}$ deletions) and 45,X/46,XY $(\mathrm{n}=40)$. No differences were found between both cytogenetic groups when two proportion Z-test analysis was applied in TS subjects with CHL, MHL or SNHL. Of 53 karyotypes with complete deletion of short arm of X-chromosome, 25 belonged to subjects with SNHL and 22 subjects with SNHL had other karyotypes $(\mathrm{P}>0.05$; OR $=0.89,95 \% \mathrm{CI}$ : 0.52-1.52).

\section{Otitis Media}

Fifty-five TS subjects (62.5\%) reported having at least one episode of otitis media, but only nine (10\%) reported a history of recurrent otitis media (defined as three or more episodes of purulent middle ear infection), three of which had been treated with myringotomy and ventilation tubes. Of the TS subjects with recurrent otitis media, only one had CHL. The presence of otitis media did not increase the chance of having $\mathrm{CHL}(\mathrm{OR}=0.34,95 \% \mathrm{CI}$ : $0.1-0.9)$ or SNHL (OR=0.70 95\% CI 0.23-2.9).

Table 4 shows the logistic regression coefficient, Wald test, and odds ratio for each of the accompanying factors for
SNHL. Overall, the binary logistic regression showed that the model fitted the data and explained a large part of the factors associated with SNHL with Nagelkerke pseudo-R2 values of 0.631 ( $-2 \log$ of likelihood: 69.27). However, only MetS $(\mathrm{P}<0.0001)$ and pterygium colli $(\mathrm{P}<0.001)$ were significant independent predictors for SNHL. The odds ratio for MetS indicates that when holding all other variables constant, a TS subject with MetS is 10.7 times more likely to have SNHL than that without MetS. Although significant, the effect of pterygium colli was much smaller than that of MetS, with a one-point increase on the nine-point MetS scale being associated with the odds of having SNHL increasing by a multiplicative factor of 5.48 .

\section{Discussion}

Individual associated factors to SNHL in women with TS such as the influence of age, ${ }^{12,13,15,16,23-25}$ chromosomal constitution $^{12,14,24,26,27}$ effect of the parental origin of the retained X-chromosome, ${ }^{6,27}$ estrogen and $\mathrm{rhGH}$ therapies, ${ }^{13-15,28,29}$ craniofacial malformations ${ }^{24,29}$ and ear infections ${ }^{13,14,25,30}$ have been systematically explored. However, the association between MetS and SNHL has not been previously evaluated. In the current study, we found a robust association between MetS or individual components of MetS and SNHL in women with TS. The prevalence of both MetS and of SNHL was higher in our adult TS cohort than that reported in other studies ${ }^{9,-12,14,23,24,29}$ This may be due to the different age ranges. ${ }^{10,11,16,23-25,31}$ We included only TS subjects $\geq 20$ years of age in our study. In studies in TS subjects with a wide age range, the prevalence of HL and

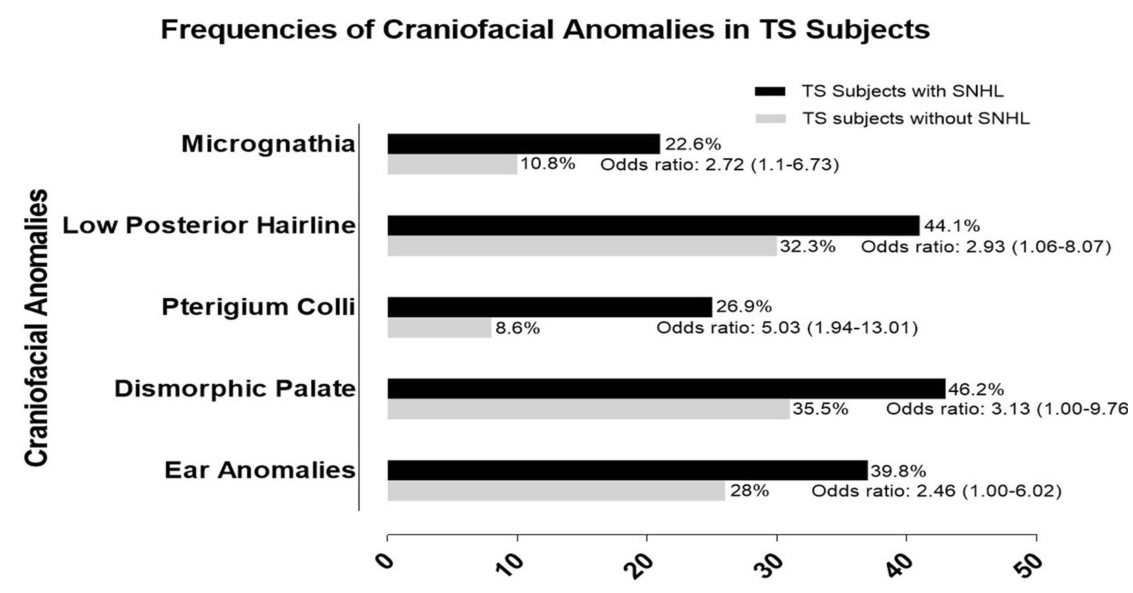

Percentages of TS Subjects with SNHL and Craniofacial Anomalies

Figure 2 Frequencies, percentages and odds ratio ( $95 \%$ confidence intervals) of the presence of anomalies in derivatives of the first and second pharyngeal arches in TS subjects with SNHL compared to those TS without SNHL.

Abbreviations: TS, Turner syndrome; SNHL, sensorineural hearing loss. 
MetS has been found to be lower. ${ }^{12,14,23,24,29}$ If TS subjects less than 20 years of age were included in our study, the prevalence of HL and of MetS could have been underestimated. On the other hand, if mostly older TS subjects ( $\geq 40$ or $\geq 50$ years of age) had been included in our study, the smaller study group could have induced an overestimation of comorbidities. In the present study, we chose a cut-off point of 30 years of age as appropriate for resolving these methodological problems. Consequently, the estimation of the odds ratios for SNHL according to MetS was adjusted for this age ( $<30$ and $\geq 30$ years). Also, other variables such as ethnic backgrounds of the individuals studied, as well as the limited health care received by our cohort rendering it more susceptible to developing both MetS and SNHL.

The aOR of SNHL in TS subjects with MetS was significantly increased than of TS subjects without MetS. Moreover, when TS subjects with MetS were subcategorized based on the number of MetS components they presented with, individuals with four components of MetS were found to have a higher aOR for SNHL than those with three components (11.05 vs 3.17). Thus, MetS appears to be an associated factor for having SNHL in adult subjects with TS. Following an increase in the number of MetS components, the chance of having SNHL also seems to increase. Our findings are reinforced by the results of a prospective longitudinal registry study in 245,000 Swedish conscripts, supporting the hypothesis that SNHL might be a clinical feature of MetS. ${ }^{32} \mathrm{We}$ found four of the five components of MetS to be independent associated factors of having SNHL in TS patients, so that each component by itself may present together with SNHL in TS. Microangiopathy is one of the proposed mechanisms for the development of SNHL, as the cochlear organ is particularly vulnerable to transient ischemia due to its highly energetic necessities and lack of collateral arterial blood flow. ${ }^{33}$ The microangiopathy hypothesis is associated with all five components of MetS. They can induce atherosclerotic changes or alter the stiffness and/or elasticity of blood vessels leading to their narrowing, and to chronic microvascular dysfunction with a decrease in blood perfusion and increased chronic cochlear damage. However, in this cross-sectional study, we were unable to elucidate if these associated factors for SNHL reflect confounding or etiological factors. Although the association of MetS and SNHL in TS could be attributable to comorbidities, our findings seem to indicate that this association likely results from progressive, additive and cumulative effects of the individual components of MetS.

Our data show that WC possesses the highest aOR for having SNHL. Being overweight/obese (BMI $\geq 25$ ) resulted in a 6-fold increase in the odds of SNHL and may be independently associated with hearing function, so that an increasing BMI, WC and WHtR may enhance the prevalence of SNHL in TS. In concordance with these findings, both a European multi-center study and a Danish study reported that high BMI and highfrequency SNHL may be associated. ${ }^{32,34}$ Similarly, among the components of MetS, hypertriglyceridemia had the second-highest aOR for SNHL in our study. Levels of TAG, TC and LDL-c, as well as atherogenic indexes (TC/HDL-c ratio, TAG/HDL-c ratio, AIP, LDL-c/ HDL-c ratio and Non-HDL-c/HDL-c ratio) were significantly increased in TS subjects with SNHL compared to non-SNHL TS individuals. Consistent with our findings, dyslipidemia has been shown to be significantly associated with chronic auditory dysfunction ${ }^{33}$ and several experimental studies in hyperlipemic animals have demonstrated profound vacuolar and structural changes of the stria vascularis and organ of Corti. ${ }^{25}$

We found a significant difference in the final height of TS subjects with and without SNHL. The association between height and SNHL has been demonstrated in two different population studies. ${ }^{31,35}$ Our findings are also in accordance with those of a previous study in which a significant correlation between short stature and SNHL was found in TS subjects. ${ }^{26}$ The Barker's hypothesis ("Thrifty Phenotype") postulates that events during fetal life may cause adult disease such as obesity, atherogenic lipid profile, hypertension, insulin resistance, T2DM and cardiovascular disease. ${ }^{36}$ These conditions are related to reduced fetal growth. Turner syndrome is one of the most frequent diseases to be accompanied by fetal growth deficiency ${ }^{37}$ and abnormal lipid and glucose profiles have been related to low birth weight and length in TS individuals. ${ }^{8}$ Thus, it is possible that the association of the components of MetS with SNHL in TS represents comorbidities and reflects confounding factors rather than etiopathogenic mechanisms.

Interestingly, in TS subjects with SNHL or CHL the presence of pterygium colli had a negative effect on hearing. A similar association between an apparent increase in the prevalence of congenital heart disease in individuals with TS and pterygium colli has been described. ${ }^{38}$ Jugular lymphatic obstruction, may lead to compression of the neck 
blood vessels, resulting in reduced blood perfusion in developing craniofacial structures. However, in contraposition to reduced blood flood, the cell cycle delay hypothesis postulates that fetal programming of the GH-IGF-1 axis may explain the association of these manifestations with short stature and SNHL. ${ }^{35}$ During embryonic development, IGF1 is crucial for several organs, including the cochlea. ${ }^{39,40}$ Additionally, in TS, low IGF-1 serum levels are related to poorer cochlear hearing function. ${ }^{25}$ A delayed cell cycle is unfavorable to tissues developing rapidly during a short time window, such as derivative structures of pharyngeal and aortic arches. Due to the study design, we were unable to evaluate this mechanism but analyzing the epigenetic marks of the genes from the GH-IGF-I axis might help clarify the association between short stature, craniofacial anomalies, MetS and SNHL in TS.

The binary logistic regression demonstrated that the model fitted the data and that the final model discriminated SNHL among our TS subjects ( $\mathrm{P}$ value $=0.9939$, HosmerLemeshow goodness of fit test). After testing multiple factors that could potentially be associated with SNHL, MetS and pterygium colli were found to be the only two significant accompanying factors to increase the likelihood of having SNHL in this model. However, pterygium colli does not have practical significance as it is an unmodifiable variable. Therefore, considering the prevalence and strength of association with SNHL, MetS was the sole modifiable factor with clinical significance. HRT use, age or complete deletion of Xp predicted a lower odds of having SNHL when run together with MetS as associated factors in a predictive binary logistic regression model for SNHL.

\section{Limitations of This Study}

This study has several limitations. First, cross-sectional design of our study precludes interpretation of temporal relationships. Our study design does not allow for the determination of any causal association between components of MetS and SNHL, and no inference can be made for the general population. This issue may be addressed with longitudinal data. In addition, because binary logistic regression analyses are correlational, inferences about the direction of causality should not be drawn. For example, SNHL may have existed prior to MetS, rather than being an effect of it. Thus, we were unable to determine if medical intervention of any component of MetS would modify the course, duration or severity of SNHL. Second, the authors of this study had no access to hearing thresholds in Venezuelan subjects. Thus, future research might want to evaluate whether the analysis of hearing thresholds in TS subjects could affect these findings. Thirdly, because of the number of statistical tests performed in this study, the nonparametric Kruskal-Wallis rank sum test followed by the Dunn test for post hoc analysis was applied to reduce the chances of obtaining a type I error (findings not shown). No modifications to the initial results were found. However, inaccurate results arising from multiple testing may still be present. The sample sizes for each group (TS subjects ( $\mathrm{n}=$ 93) and reference group $(n=57))$ were relatively small, resulting in even smaller sample sizes when broken down into groups for analysis. This may cause inaccurate inferences made from associated statistical tests. It may be unnecessarily rigorous to perform so when there is a priori evidence of some associations being tested. Thus, we suggest these associations be further investigated and replicated in other cohorts. Four, the mechanisms evaluating how MetS could cause SNHL were not analyzed in this study and we recognize that the relationship between $\mathrm{HL}$ and most of the factors studied, so far, is uncertain and insufficiently documented. Further studies will be needed to explain the underlying pathological mechanism connecting MetS and SNHL, for example, the role of obesity-induced low-grade inflammation and/or its related adipocytokines on hearing thresholds. Lastly, the lack of data at birth, family history and the degree of exposure to treatment (eg antibiotics (aminoglycosides), diuretics (furosemide) or exposure to noise in our cohort are other limitations, which should be controlled for both in future retrospective and prospective studies, which should be controlled for both in future retrospective and prospective studies.

\section{Conclusion}

In summary, SNHL was the most frequent type of hearing loss found in our adult TS cohort. Women with TS and SNHL were significantly shorter and had a higher weight, BMI, WC, WHR, WHtR and blood pressures than nonSNHL subjects. The prevalence of MetS was higher in SNHL than in Non-SNHL TS subjects and MetS and its individual components were associated factors for having SNHL in our cohort. In addition, the proportion of external craniofacial signs was significantly larger in TS subjects with SNHL. Neither recurrent otitis media, nor HRT or chromosomal constitution were associated to SNHL in our TS cohort. Given the connection established between SNHL and MetS in TS subjects, a reduction in the number and severity of the components of MetS might potentially contribute to reducing the progression of SNHL at younger 
ages. Further studies will be needed to explain the underlying pathological mechanisms connecting MetS and SNHL.

\section{Statement of Ethics}

The study protocol was reviewed and approved by the Ethics Committee's for Research in Human Subjects of the two participating institutions. The ethical principles of the 1964 Declaration of Helsinki for medical research were adhered to throughout this research. The procedures and possible discomfort/risks were fully explained to all participating subjects before beginning the study. Each then voluntarily decided to take part in the study and approved their participation and signed an informed written consent form in front of a witness. Subjects were provided with the option to withdraw their participation in the study at any time without consequence.

\section{Acknowledgments}

We are extremely grateful to all women with Turner syndrome who took part in this study. We acknowledge the contribution of Ecuadorian Foundation in Support of Turner Syndrome. The authors would like to thank the Consejo de Desarrollo Científico, Humanístico y Tecnológico (CONDES) from University of Zulia, Maracaibo, Venezuela who provided assistance with the program. This study was supported by the Academie de Recherche et D'Enseignement Superieur of Belgique (grant number 2016-157E); and Training Research Commission, General Directorate of Research and Postgraduate from Central University of Ecuador (grant number cif3-cv-fcb-2).

\section{Disclosure}

The authors declare that they have nothing to disclose and there is no conflict of interest that could be perceived as prejudicing the impartiality of the research reported.

\section{References}

1. Álvarez-Nava F, Soto M, Sánchez MA, et al. Molecular analysis in Turner syndrome. J Pediatr. 2003;142(3):336-340. doi:10.1067/ mpd.2003.95

2. Gravholt $\mathrm{CH}$, Andersen NH, Conway GS, et al. Clinical practice guidelines for the care of girls and women with Turner syndrome: proceedings from the 2016 cincinnati international turner syndrome meeting. Eur J Endocrinol. 2017;177(3):G1-G70. doi:10.1530/EJE17-0430

3. Stochholm K, Juul S, Juel K, et al. Prevalence, incidence, diagnostic delay, and mortality in Turner syndrome. J Clin Endocrinol Metab. 2006;91:3897-3902. doi:10.1210/jc.2006-0558
4. Schoemaker MJ, Swerdlow AJ, Higgins CD, et al. Mortality in women with turner syndrome in Great Britain: a national cohort study. J Clin Endocrinol Metab. 2008;93:4735-4742. doi:10.1210/ jc. 2008-1049

5. Elsheikh M, Dunger DB, Conway GS, et al. Turner's syndrome in adulthood. Endocr Rev. 2002;23:120-140. doi:10.1210/ edrv.23.1.0457

6. Álvarez-Nava F, Lanes R, Quintero JM, et al. Effect of the parental origin of the $\mathrm{X}$-chromosome on the clinical features, associated complications, the two-year-response to growth hormone ( $\mathrm{rhGH})$ and the biochemical profile in patients with Turner syndrome. Int J Pediatr Endocrinol. 2013;2013(1):10. doi:10.1186/1687-98562013-10

7. Alberti KGMMG, Zimmet P, Shaw J, et al. The metabolic syndrome a new world-wide definition. A consensus statement from the International Diabetes Federation. Lancet. 2005;336 (9491):1059-1062. doi:10.1111/j.1464-5491.2006.01858.x

8. Baldin AD, Siviero-Miachon AA, Fabbri T, et al. Turner syndrome and metabolic derangements: another example of fetal programming. Early Hum Dev. 2012;88:99-102. doi:10.1016/j. earlhumdev.2011.07.014

9. Lebenthal Y, Levy S, Sofrin-Drucker E, et al. The natural history of metabolic comorbidities in Turner syndrome from childhood to early adulthood: comparison between 45,X monosomy and other karyotypes. Front Endocrinol (Lausanne). 2018;9:27. doi:10.3389/ fendo.2018.00027

10. Landin-Wilhelmsen K, Bryman I, Wilhelmsen L. Cardiac malformations and hypertension, but not metabolic risk factors, are common in Turner syndrome. J Clin Endocrinol Metabol. 2001;86:4166-4170. doi:10.1210/jcem.86.9.7818

11. Calcaterra V, Brambilla P, Maffè GC, et al. Metabolic syndrome in Turner syndrome and relation between body composition and clinical, genetic, and ultrasonographic characteristics. Metab Syndr Relat Disord. 2014;12:159-164. doi:10.1089/met.2013.0075

12. Hederstierna C, Hultcrantz M, Rosenhall U. A longitudinal study of hearing decline in women with Turner syndrome. Acta Otolaryngol. 2009;129:1434-1441. doi:10.3109/00016480902741962

13. Ros C, Tercero A, Alobid I, et al. Hearing loss in adult women with Turner's syndrome and other congenital hypogonadisms. Gynecol Endocrinol. 2014;30(2):111-116. doi:10.3109/0951359 0.2013 .856002

14. Verver EJ, Freriks K, Sas TC, et al. Karyotype-specific ear and hearing problems in young adults with turner syndrome and the effects of oxandrolone treatment. Otol Neurotol. 2014;35:1577-1584. doi:10.1097/MAO.0000000000000406

15. Silva Oliveira C, Mendonça Ribeiro F, Lago R, et al. Audiological abnormalities in patients with Turner syndrome. Am J Audiol. 2013;22:226-232. doi:10.1044/1059-0889(2013/11-0027)

16. Parkin M, Walker P. Hearing loss in Turner syndrome. Int J Pediatr Otorhinolaryngol. 2009;73:243-247. doi:10.1016/j. ijporl.2008.10.0127

17. Han X, Wang Z, Wang J, et al. Metabolic syndrome is associated with hearing loss among a middle-aged and older Chinese population: a cross-sectional study. Ann Med. 2018;50:587-595. doi:10.1080/07853890.2018.1469786

18. Chien CY, Tai SY, Wang LF, et al. Metabolic syndrome increases the risk of sudden sensorineural hearing loss in Taiwan: a case-control study. Otolaryngol Head Neck Surg. 2015;153:105-111. doi:10.1177/ 0194599815575713

19. Sun YS, Fang WH, Kao TW, et al. Components of metabolic syndrome as risk factors for hearing threshold shifts. PLoS One. 2015;10:e134388. doi:10.1371/journal.pone.0134388

20. Shim HS, Shin HJ, Kim MG, et al. Metabolic syndrome is associated with hearing disturbance. Acta Otolaryngol. 2019;139:42-47. doi:10.1080/00016489.2018.1539515 
21. Aghazadeh-Attari J, Mansorian B, Mirza-Aghazadeh-Attari M, et al. Association between metabolic syndrome and sensorineural hearing loss: a cross-sectional study of 11,114 participants. Diabetes Metab Syndr Obes. 2017;6:459-465. doi:10.2147/DMSO.S150893

22. Beckman A, Conway GS, Cadge B. Audiological features of Turner's syndrome in adults. Int $J$ Audiol. 2004;43:533-544. doi:10.1080/ 14992020400050068

23. King KA, Makishima T, Zalewski CK, et al. Analysis of auditory phenotype and karyotype in 200 females with Turner syndrome. Ear Hear. 2007;28:831-841. doi:10.1097/AUD.0b013e318157677f

24. Hultcrantz M, Sylvén L. Turner's syndrome and hearing disorders in females aged 16-34. Hear Res. 1997;103:69-74. doi:10.1016/S03785955(96)00165-7

25. Barrenäs ML, Bratthall $\AA$, Dahlgren J. The association between short stature and sensorineural hearing loss. Hear Res. 2005;205:123-130. doi:10.1016/j.heares.2005.03.019

26. Ostberg JE, Beckman A, Cadge B, et al. Oestrogen deficiency and growth hormone treatment in childhood are not associated with hearing in adults with Turner syndrome. Horm Res. 2004;62:182-186. doi:10.1159/000080888

27. Morimoto N, Tanaka T, Taiji H, et al. Hearing loss in Turner syndrome. $J$ Pediatr. 2006;149:697-701. doi:10.1016/j. jpeds.2006.06.071

28. Hamelin CE, Anglin G, Quigley CA, et al. Genomic imprinting in Turner syndrome: effects on response to growth hormone and on risk of sensorineural hearing loss. $J$ Clin Endocrinol Metab. 2006;91:3002-3010. doi:10.1210/jc.2006-0490

29. Stenberg AE1, Nylén O, Windh M, Hultcrantz M. Otological problems in children with Turner's syndrome. Hear Res. 1998;124:85-90. doi:10.1016/S0378-5955(98)00113-0

30. Barrenäs ML, Landin-Wilhelmsen K, Hanson C. Ear and hearing in relation to genotype and growth in Turner syndrome. Hear Res. 2000;144:21-28. doi:10.1016/S0378-5955(00)00040-X

31. Barrenäs ML, Jonsson B, Tuvemo T, et al. High risk of sensorineural hearing loss in men born small for gestational age with and without obesity or height catch-up growth: a prospective longitudinal register study on birth size in 245,000 swedish conscripts. J Clin Endocrinol Metab. 2005;90:4452-4456. doi:10.1210/jc.2005-0385
32. Jung SY, Shim HS, Hah YM, et al. Association of metabolic syndrome with sudden sensorineural hearing loss. JAMA Otolaryngol Head Neck Surg. 2018;144:308-314. doi:10.1001/jamaoto.2017.3144

33. Fransen E, Topsakal V, Hendrickx JJ, et al. Occupational noise, smoking, and a high body mass index are risk factors for age-related hearing impairment and moderate alcohol consumption is protective: a European population-based multicenter study. $J$ Assoc Res Otolaryngol. 2008;9:264-276. doi:10.1007/s10162-008-0123-1

34. Satar B, Ozkaptan Y, Sürücü HS, et al. Ultrastructural effects of hypercholesterolemia on the cochlea. Otol Neurotol. 2001;22:786-789. doi:10.1097/00129492-200111000-00012

35. Barker D. The fetal and infant origins of adult disease. The womb may be more important than the home. BMJ. 1990;301:1111. doi:10.1136/bmj.301.6761.1111

36. Wisniewski A, Milde K, Stupnicki R, et al. Weight deficit at birth and Turner's syndrome. J Pediatr Endocrinol Metab. 2007;20:607-613. doi:10.1515/JPEM.2007.20.5.607

37. Loscalzo ML, Van PL, Ho VB, et al. Association between fetal lymphedema and congenital cardiovascular defects in Turner syndrome. Pediatrics. 2005;115(3):732-735. doi:10.1542/peds.20041369

38. Rodríguez-de la Rosa L, Lassaletta L, Calvino M, et al. The role of insulin-like growth factor 1 in the progression of age-related hearing loss. Front Aging Neurosci. 2017;9:411. doi:10.3389/ fnagi.2017.00411;

39. Morales-Garcia JA, Vigil P, Diaz-Casares A, et al. Trophic effects of insulin-like growth factor-I (IGF-I) in the inner ear. Hear Res. 2004;196:19-25. doi:10.1016/j.heares.2003.12.022

40. Varela-Nieto I, Morales-Garcia JA, Vigil P, et al.Trophic effects of insulin-like growth factor-I (IGF-I) in the inner ear. Hear Res. 2004;196(1-2):19-25. doi:10.1016/j.heares.2003.12.022
The Application of Clinical Genetics

\section{Publish your work in this journal}

The Application of Clinical Genetics is an international, peerreviewed open access journal that welcomes laboratory and clinical findings in the field of human genetics. Specific topics include: Population genetics; Functional genetics; Natural history of genetic disease; Management of genetic disease; Mechanisms of genetic disease;

\section{Dovepress}

Counselling and ethical issues; Animal models; Pharmacogenetics; Prenatal diagnosis; Dysmorphology. The manuscript management system is completely online and includes a very quick and fair peerreview system, which is all easy to use. Visit http://www.dovepress. com/testimonials.php to read real quotes from published authors. 